\title{
Titanate Nanotubes as New Nanostrutured Catalyst for Depolymerization of PET by Glycolysis Reaction
}

\author{
Gabrielle Ritter Lima ${ }^{a *}$, Wesley Formentin Monteiro ${ }^{b}$, Rosane Ligabue ${ }^{b}$,
}

\section{Ruth Marlene Campomanes Santana ${ }^{a}$}

\author{
${ }^{a}$ Programa de Pós Graduação em Engenharia de Minas, Metalúrgica e Materiais - PPGE3M, \\ Universidade Federal do Rio Grande do Sul, UFRGS, Porto Alegre, RS, Brazil \\ ${ }^{b}$ Programa de Pós Graduação em Engenharia e Tecnologia de Materiais - PGETEMA, Pontifícia \\ Universidade Católica do Rio Grande do Sul - PUCRS, Porto Alegre, RS, Brazil
}

Received: July 12, 2017; Revised: September 22, 2017; Accepted: October 05, 2017

\begin{abstract}
The final destination of PET packaging is creating economic and environmental concerns. One of the alternatives to minimize this problem would be making use of chemical recycling of this material through glycolysis with the aim to produce bis(hydroxiethyl) terephthalate, BHET monomer. This reaction is well known, but it still presents problems as BHET purity since it makes necessary the development of new catalysts highly selective. In this context, the present work studied the catalytic activity of a nanostructured material, titanate nanotubes (TNT), and compared it to a commercial catalyst (zinc acetate), which is the most used for this glycolysis reaction according to literature researches, and analyzed the influence of PET type (virgin and post-consumer) in the depolymerization for reaction times of 2, 3 and 4 hours. Using TNT as catalyst, BHET production yield and values of turnover number for the evaluated reaction times were higher than the results using $\mathrm{Zn}(\mathrm{OAc})_{2}$ for virgin PET, proving itself as a promising catalyst.
\end{abstract}

Keywords: chemical recycling, glycolysis, nanostructure catalysts, titanate nanotubes

\section{Introduction}

Poly(ethylene terephthalate), PET, is a commodity polymer of great importance due to its excellent physical and chemical properties ${ }^{1}$. PET also offers excellent barrier property and low weight compared to glass packaging ${ }^{2}$. Therefore, this polymer is especially used in the packing industry. However, PET has a high global production and, consequently, consumption and non-biodegradability makes the disposal of post-consumer PET waste a problem as far as economic and environmental considerations are concerned. Among the various possibilities to mitigate this problem, the chemical recycling process would be the best alternative for this material. This process is in accordance with the principles of sustainable development because it leads to the formation of raw materials (monomers) used for producing $\mathrm{PET}^{3}$. Chemical recycling by glycolysis is one of de most used recycling types. It can be described as a depolymerization process by transesterification between PET ester groups and a diol (generally ethylene glycol, EG) ${ }^{4,5}$, allowing obtaining of bis(hydroxyethyl) terephthalate $(\mathrm{BHET})^{6}$ as main depolymerization product (Figure 1).

This reaction depends on some variables such as temperature, molar ratio EG/PET, glycolysis time and amount of catalyst ${ }^{3}$. Among these variables, the catalyst type can dramatically influence the reaction conditions such

*e-mail: gabrielleritterlima@gmail.com as low temperature and reaction time. Recently various transition metal-based catalysts with good catalytic activities on PET glycolysis have been described in literature, the most used among them being zinc acetate ${ }^{7,8}$, but they have limitations such as difficult synthesis of the catalyst ${ }^{1,9}$ or catalyst toxicity or corrosivity, resulting in pollution ${ }^{10}$. Thus, the search for new highly selective catalysts under mild conditions is required ${ }^{3}$.

Among the materials with potential application for catalysis are nanomaterials, such as nanotubes, nanoribbons, nanowires that exhibit unique properties, such as, changes in chemical reactivity and electrical conductivity ${ }^{11,12}$, which, combined, are not found in conventional materials ${ }^{13}$. Recent studies showed that titanate nanotubes have Brönsted and Lewis acid sites $^{14}$ formed from lattice distortion due to the scrolling of titanate nanotubes layers. This property makes this nanomaterial an effective catalysts in various applications as in $\mathrm{CO}_{2}$ conversion ${ }^{15,16}$, biodiesel synthesis $^{17-19}$, styrene epoxidation ${ }^{20}$, oxidesulfurization process $^{21}$, nanocomposite polymer ${ }^{22}$ among others. However, until present moment, there aren't studies that show the catalytic activity of TNT in glycolysis reactions, representing a yet unexploited area.

In this context, this study aims to evaluate the catalytic activity of TNT compared with zinc acetate, at different reaction times and to evaluate the influence of PET source (virgin and post-consumer bottle-grade) in the PET depolymerization. 


\section{Material and Methods}

The materials used for the production of titanate nanotubes (TNT) and glycolysis reactions were: sodium hydroxide (99\% Vetec), titanium dioxide ( $98 \%$ anatase phase, JB Química), zinc acetate, (98\%, Fmaia), ethylene glycol (99.5\%, Dinâmica), virgin PET (Rhopet S-80 - Rhodia Ster/ Mossi and Ghisolfi Group) and post-consumer bottle-grade PET. All reactants were used as received. The filters used to the first and second filtration were Unifil C42 (1-2 $\mu \mathrm{m})$.

\subsection{Titanate nanotubes synthesis}

The TNT were synthesized based on the hydrothermal method adapted from Kasuga et al. (1998) ${ }^{23}$ according to the following procedure: in a beaker was weighted $1.5 \mathrm{~g}$ of titanium dioxide and added $120 \mathrm{~mL}$ of $10 \mathrm{M} \mathrm{NaOH}$ aqueous solution. The mixture was kept under magnetic stirring for 30 minutes at room temperature. After that, the mixture was added to a stainless steel autoclave reactor and remained reacting at $130-140^{\circ} \mathrm{C}$ for $72 \mathrm{~h}$. Finally the formed precipitate was washed with distilled water and centrifuged several times until the $\mathrm{pH}$ of washing water reached about 7.

\subsection{PET glycolysis}

For PET depolymerization reactions by glycolysis, it was used virgin PET in pellets $(2,85 \times 2,60 \mathrm{~mm})$. In the case of post-consumer PET bottles, these were from carbonated drinks, exclusively of soda, and transparent, in order to avoid variations relating to colorants and the polymer crystallinity. These bodies of PET bottles were separated from other materials that are used to compose the bottles, like the covers (PP) and labels (LDPE or PP). They were then washed, dried, manually cutted into dimensions of about $5 \times 5 \mathrm{~mm}$ and again washed and dried, to remove any impurities from the cutting step.

The PET glycolysis was optimized based on literature ${ }^{24,25}$ where $15 \mathrm{~g}$ PET, $60 \mathrm{~g}$ EG and $50 \mathrm{mg}$ of catalyst were added to a $500 \mathrm{~mL}$ round-bottom three-naked flask equipped with magnetic stirrer, thermometer and reflux condenser. The reactions were carried out at $196^{\circ} \mathrm{C}$ since it is the boiling temperature of EG. The glycolysis reaction was carried out at different times (2, 3 and 4 hours) and after it was added approximately $300 \mathrm{~mL}$ of boiling water into flask so the products formed were dissolved. Then it was filtered under reduced pressure and the filtrate was stored at $4-10^{\circ} \mathrm{C}$ for $72 \mathrm{~h}$ to forming the BHET crystals. The separation and reuse of the catalyst weren't aim of this work. After, the BHET crystals were filtered under reduced pressure using G4 sintered glass funnel, to ensure that catalysts used in the reaction were retained during filtration. The white BHET crystals were dried at $60^{\circ} \mathrm{C}$ for 24 hours. The influence of reaction time was then evaluated in PET conversion results and yield of BHET. The conversion (C) of PET in glycolysis reactions was calculated based on the Eq. (1):

$$
C=\frac{W_{i}-W_{f}}{W_{i}} \times 100
$$

where $W_{i}$ represents the initial weight of PET and $W_{f}$ represents the weight of undepolymerized PET.

The molar yield (X) of BHET produced by PET depolymerization was calculated according to Eq. (2):

$$
X=\frac{W_{B H E T, f} / M W_{B H E T}}{W_{P E T, i} / M W_{P E T}} \times 100
$$

where $W_{B H E T, f}$ represents the final weight of BHET, $M W_{B H E T}$ represents the molar weight of BHET (254 g.mol $\left.{ }^{-1}\right), W_{P E T, i}$ represents the initial weight of PET used in the reaction and $M W_{P E T}$ represents the molecular weight of the repeat unit of PET chain (192 g. $\left.\mathrm{mol}^{-1}\right)$.

The turn over number (TON) of the used catalyst was calculated according to Eq. (3):

$$
T O N=\frac{\text { mols of product }}{\text { mols of catalyst }}
$$

And the turn over frequency (TOF) of the catalyst was calculated based on Eq. (4):

$$
T O F=\frac{T O N}{\text { time }(h)}
$$

\subsection{Characterization}

\subsubsection{Titanate nanotubes (TNT)}

\section{Scanning Electron Microscopy (SEM)}

TNT morphology was analyzed by field emission scanning electron microscopy (FEI Inspect F50) in SE mode (secondary electron beam). The samples were analyzed as powder using gold film deposited on the surface by ion-sputter.

Transmission Electron Microscopy (TEM)

The evaluation of the internal structure and numbers of rolled multilayer lamellar walls of TNT was made using transmission electron microscopy (FEI Tecnai G2 T20) using cupper grids with carbon film (300 mesh). The measures of TNT dimensions were obtained by TEM analysis used Image $J$ software (number of measurements $=25$ ).

X-Ray Diffraction (XRD)

The characterization of the crystalline structure of TNT was made by X-ray diffraction analysis (Shimadzu XRD $7000)$ using radiation $\mathrm{K} \alpha$ of the copper $(\lambda=1.542 \AA)$, voltage $40 \mathrm{kV}, 30 \mathrm{~mA}$, scanning between $5^{\circ}-70^{\circ} 2 \theta$ and scan speed of $0.02 \% \mathrm{~min}$. In all analysis TNT was in powder form.

\subsubsection{PET glycolysis}

Differential Scanning Calorimetry (DSC)

BHET formed by PET depolymerization reactions were characterized by thermal analysis of Differential Scanning Calorimetry (DSC) in a calorimeter, Model Q20 from TA Instruments in the range from $45^{\circ} \mathrm{C}$ to $270^{\circ} \mathrm{C}$ at a heating 


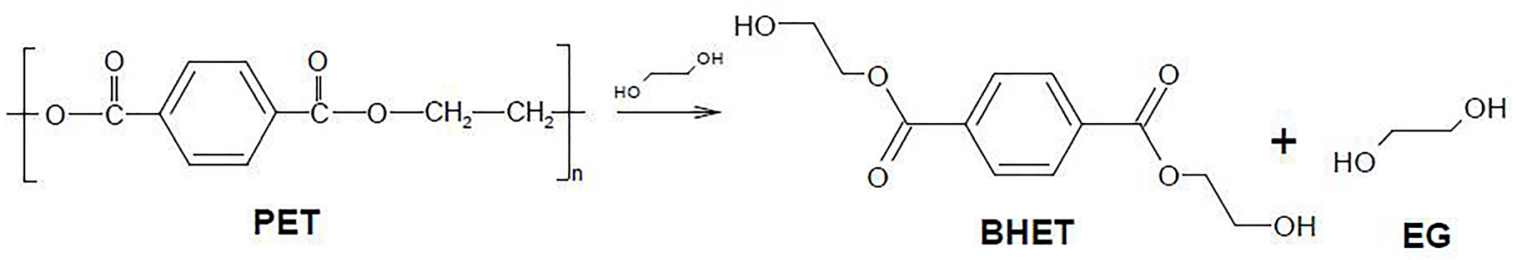

Figure 1. Reaction of PET depolymerization by glycolysis.

rate of $10^{\circ} \mathrm{C} \cdot \mathrm{min}^{-1}$, under inert atmosphere of $\mathrm{N}_{2}$. Melting enthalpy analyzes were made in triplicate.

Thermogravimetric Analysis (TGA)

The thermal stability of starting virgin and post-consumer PET and BHET formed by PET depolymerization reactions were characterized by thermogravimetric analysis (TGA) in a SDT equipment, Model Q600 from TA Instruments in the range from $50^{\circ} \mathrm{C}$ to $800^{\circ} \mathrm{C}$ at a heating rate $20^{\circ} \mathrm{C} \cdot \mathrm{min}^{-1}$, under inert atmosphere of $\mathrm{N}_{2}$.

Nuclear Magnetic Resonance Spectroscopy (NMR)

In order to confirm the production of BHET, ${ }^{1} \mathrm{H}-\mathrm{NMR}$ and ${ }^{13} \mathrm{C}-\mathrm{NMR}$ were recorded on a Bruker Ascend 400 NMR spectrometer operating at $400 \mathrm{MHz}$ in deuterated DMSO solution.

\section{Results and Discussion}

\subsection{Titanate Nanotubes (TNT)}

The formation of titanate nanotubes (TNT) was confirmed by SEM and TEM analysis (Figures 2a and 2b, respectively). As expected these nanotubes have a tubular morphology but very crowded. This agglomeration clearly observed by the SEM image (Figure 2a) can occur due to some effect on the drying process. Figure $2 b$ shows TEM image of the TNT, where it is possible to observe that the nanotubes are formed by winding at least three titanates multilayer lamellar walls and have an external diameter of $8.6 \mathrm{~nm} \pm 1.4 \mathrm{~nm}$ and presents wall thickness of $0.6 \mathrm{~nm} \pm 0.1 \mathrm{~nm}$ (calculated using Image $J$ software in Fig. 2). These results are in agreement with those found by Monteiro et al. ${ }^{22}$.

Figures 3 shows the diffraction pattern obtained of $\mathrm{TiO}_{2}$ precursor (anatase phase) and the characteristics peaks are situated at $2 \theta=25^{\circ}(101), 38^{\circ}(004), 48^{\circ}(200), 53^{\circ}(106)$ and $62^{\circ}(215)$ in agreement with the assignments described in literature ${ }^{26,27}$. In relation to TNT diffractogram, this shows disappearance of the fine peak situated at $2 \theta=25^{\circ}$ (101), main peak of anatase phase and appearance of signs of lamellar titanates, $2 \theta=24^{\circ}(110), 28^{\circ}(211), 48^{\circ}(020)$ and $62^{\circ}(422)^{28,29}$ and the peak at around $2 \theta=10^{\circ}$ assigned to the interlayer distance ${ }^{30}$.

\subsection{BHET characterization}

In order to confirm the BHET production as the main product of PET glycolysis obtained at different times and with catalysts TNT or $\mathrm{Zn}(\mathrm{OAC}){ }_{2}$, DSC, TGA, ${ }^{1} \mathrm{H}$ and ${ }^{13} \mathrm{C}-\mathrm{NMR}$ analysis were performed.

Figure 4 shows the DSC curves of the main product of glycolysis from virgin (Figure 4a) and post-consumer PET (Figure 4b), which has an endothermic peak located at $110^{\circ} \mathrm{C}$ corresponding to the melting point of $\mathrm{BHET}^{10}$. Moreover, there are not observed peaks corresponding to dimers and/ or oligomers $\left(\sim 170^{\circ} \mathrm{C}\right)^{31}$ and unreacted residual PET $(255$ -
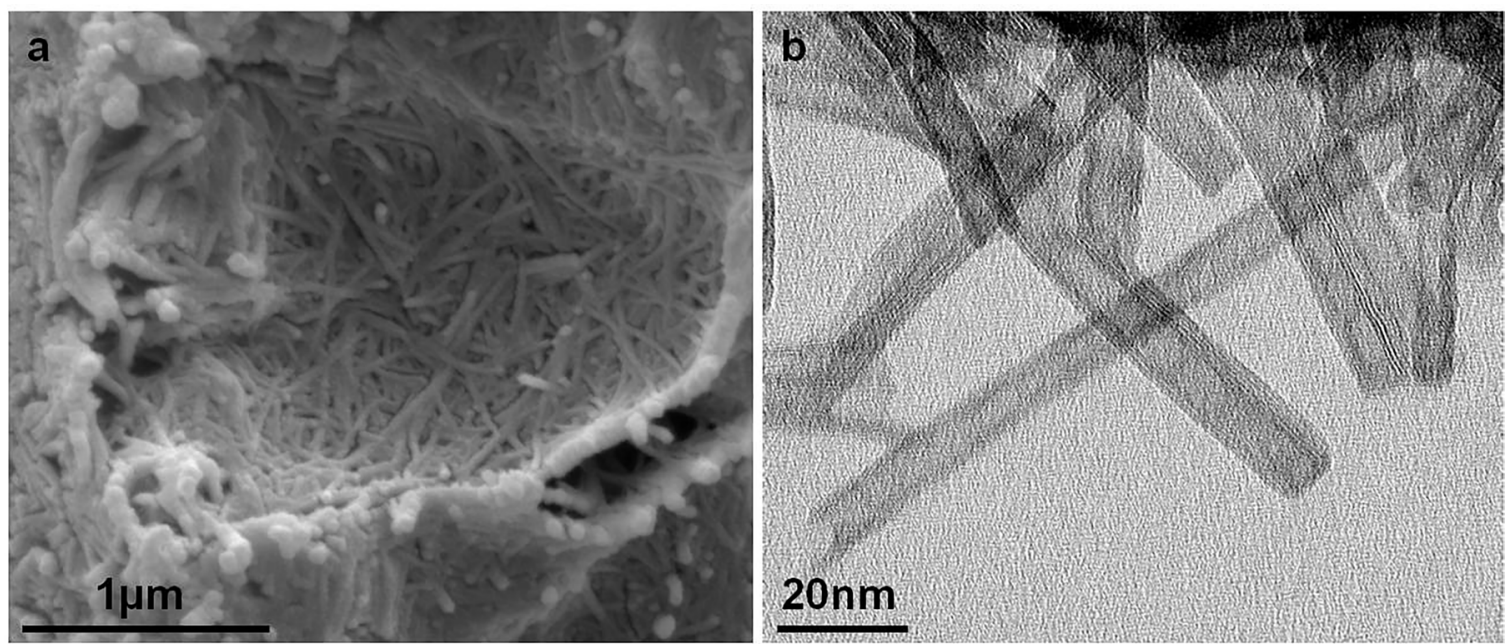

Figure 2. Images of TNT obtained by a) SEM (magnification of 100k) and b) TEM (magnification of 410K). 


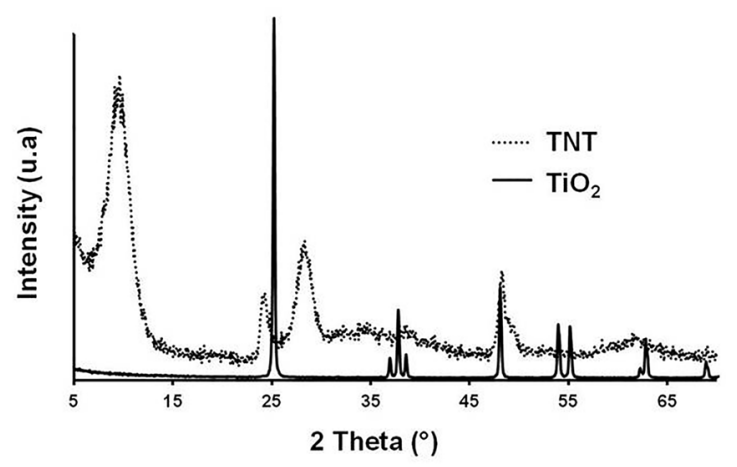

Figure 3. XRD results of $\mathrm{TiO}_{2}$ and TNT.

$\left.265^{\circ} \mathrm{C}\right)^{32}$, indicating that both catalysts were efficient in virgin and post-consumer PET glycolysis.

The melting enthalpy $(\Delta \mathrm{Hm})$ for the glycolysis product (BHET) obtained by the use of virgin (Figure 5a) and postconsumer PET (Figure 5b) and catalyzed by TNT or $\mathrm{Zn}(\mathrm{OAc})_{2}$ are shown in Figure 5. As noted, regardless the type of PET or the catalyst used on glycolysis reaction, BHET melting enthalpy values showed no significant difference, indicating that BHET purity in different reaction conditions is similar.

Thermogravimetric analysis (Figure 6) of the main product of glycolysis of virgin PET (Figure 6a) and post-consumer
PET (Figure 6b) shows small weight loss differences in the degradation first stage that occurs around $250^{\circ} \mathrm{C}$ (degradation of BHET product). Followed by a second stage which starts at about $400^{\circ} \mathrm{C}$ that it is related to PET degradation formed by thermal polymerization of BHET during analysis ${ }^{31}$. Figure $6 \mathrm{c}$ and $6 \mathrm{~d}$ shows in detail the first degradation stage for BHET obtained from virgin and post-consumer PET, respectively. There are included in Figure 6 the degradation curves of virgin PET and post-consumer PET. Degradation of virgin PET (Figure 6a) occurs at one single step starting at approximately $400^{\circ} \mathrm{C}$, as well as degradation of postconsumer PET (Figure 6b).

Table 1 shows the initial weight loss temperature, Ti 5\% w. 1., (considered the temperature when the sample loses 5\% of its weight) of all samples, \% weight loss for the first and second stages of BHET and PET degradation, respectively, and also $\%$ of ash content. Results of degradation temperature at $5 \%$ weight loss showed, with small difference, that BHET obtained using $\mathrm{Zn}(\mathrm{OAc})_{2}$ are more thermally stable for both virgin and post-consumer PET, because it is observed the need of higher temperatures to initiate degradation.

Table 1 also shows a weight loss difference in the first stage of degradation, with the TNT catalyst samples being smaller (about 25\%) than those with $\mathrm{Zn}(\mathrm{OAc})_{2}$ (about 30\%) for BHET obtained from virgin PET. For BHET obtained from
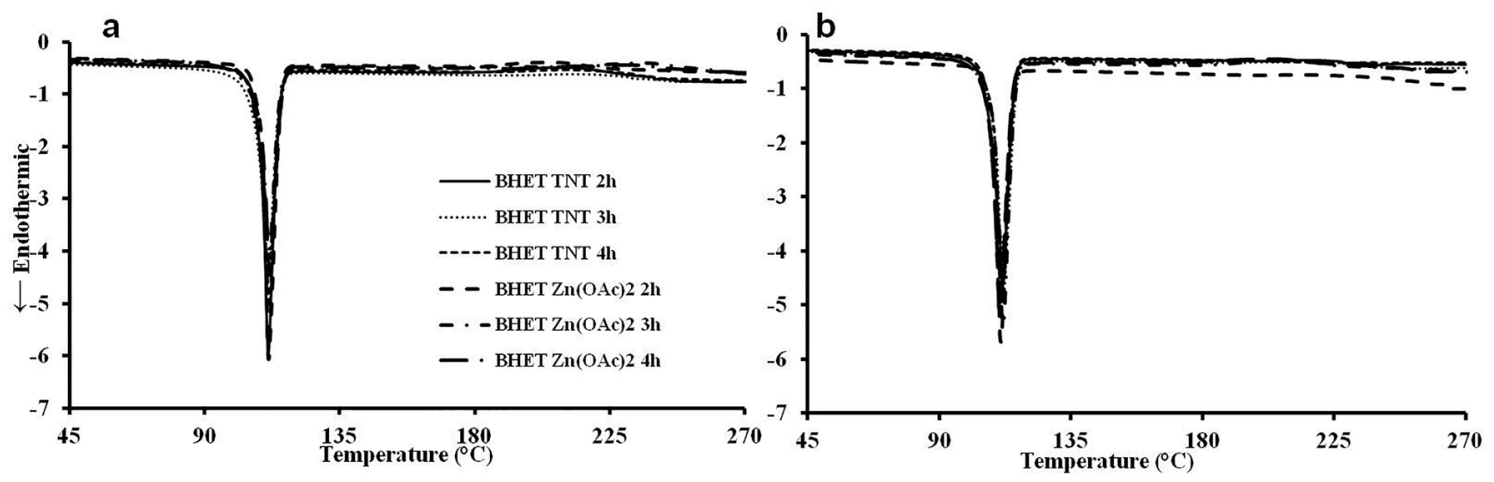

Figure 4. DSC curves of the BHET obtained from: a) virgin PET and b) post-consumer PET.

a

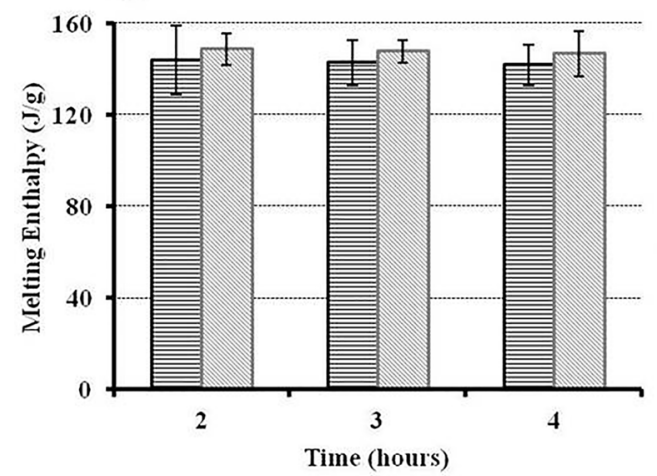

b

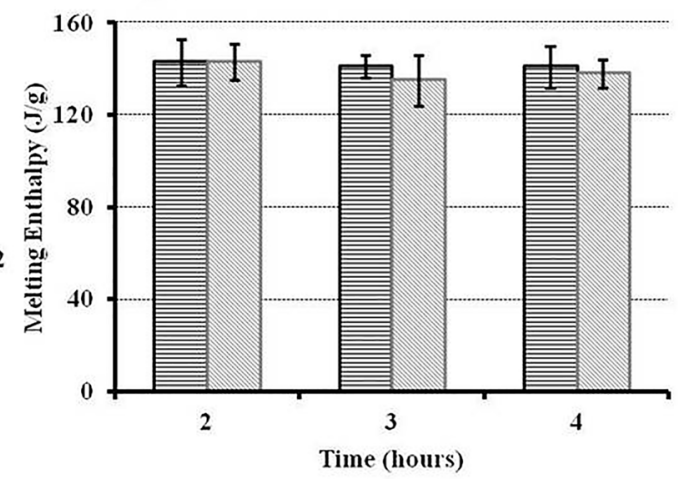

Figure 5. Enthalpies of fusion for BHET from: a) virgin PET and b) post-consumer PET. 
post-consumer PET, weight loss of samples using both TNT and $\mathrm{Zn}(\mathrm{OAc})$, as catalyst remained the same (about 25\%). This results are in agreement with what has been showed previously about the purity of the BHET being unaffected by the use of different catalysts.

Samples from virgin PET and the starting material showed little difference in ash content (about 10-12\%). Furthermore, to post-consumer, ash content presented higher difference (about 6-13\%) that can be associated with impurities, such as additives used during processing of $\mathrm{PET}^{33,34}$.

Accordingly to Geng et al..$^{35}$ and Imran et al. ${ }^{31}$ weight loss about $20 \%$ at $250^{\circ} \mathrm{C}$ on the first step refers to the thermal decomposition of BHET dimers, and the second weight loss about $70 \%$ at $400^{\circ} \mathrm{C}$ refers to the thermal decomposition of the PET produced from the BHET dimer thermal repolymerization during the TGA process. Differently from these results, the TGA analysis in this work showed the first step of degradation refers to basically BHET monomer. This result is corroborated by the results of NMR (Figure 7 and 8), where no peaks were observed regarding BHET dimers. The NMR spectra presented signals referring only to BHET monomer.

The chemical structure of BHET was analyzed by nuclear magnetic resonance (NMR) spectroscopy. The ${ }^{1} \mathrm{H}-\mathrm{NMR}$ spectrum of BHET is shown in Figure 7 with chemical structure illustration.

The signs labelled 1, 2, 3 and 4 (Figure 7) are assigned the protons of the aromatic ring $\left(\delta_{\mathrm{H}}=8.1 \mathrm{ppm}, \mathrm{s}, 4 \mathrm{H}\right)$, hydroxyl groups $\left(\delta_{\mathrm{H}}=4.95 \mathrm{ppm}, \mathrm{t}, 2 \mathrm{H}\right)$, methylenes $\left(-\mathrm{CH}_{2}-\right)$ adjacent to the $-\mathrm{OH}$ groups $\left(\delta_{\mathrm{H}}=3.73 \mathrm{ppm}, \mathrm{m}, 4 \mathrm{H}\right)$, methylenes
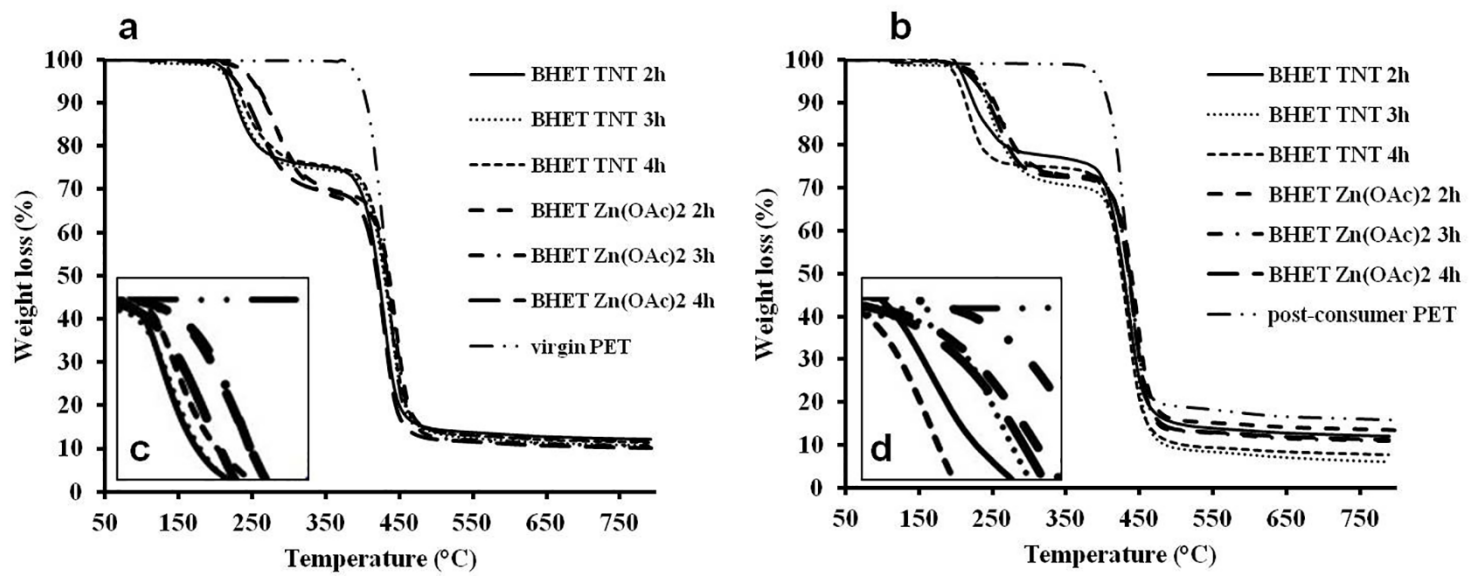

Figure 6. TGA curves for the starting material and BHET obtained from: a) virgin PET, b) post-consumer PET, c) increased image for first stage weight loss for BHET from virgin PET and d) increased image for first stage weight loss for BHET from post-consumer PET.

Table 1. Degradation temperature at $5 \%$ weight loss, weight loss for the $1^{\circ}$ and $2^{\circ}$ degradation stages, ash content of TGA results obtained for both starting material and BHET from virgin and post-consumer PET for different reaction times.

\begin{tabular}{|c|c|c|c|c|c|c|}
\hline & Samples & Time (h) & $\mathrm{Ti}\left({ }^{\circ} \mathrm{C}\right) 5 \%$ w. 1. & W. $\mathrm{L}_{1}(\%)$ & W. $\mathrm{L}_{2}(\%)$ & Ash content $(\%)$ \\
\hline \multirow[t]{3}{*}{ Starting virgin PET } & & - & 396.0 & 84.9 & - & 11.0 \\
\hline & & 2 & 218.1 & 23.0 & 61.2 & 12.2 \\
\hline & TNT & 3 & 218.1 & 23.5 & 61.5 & 10.7 \\
\hline \multirow{4}{*}{ Virgin PET } & & 4 & 227.2 & 24.0 & 62.4 & 11.4 \\
\hline & & 2 & 228.7 & 30.1 & 57.2 & 10.2 \\
\hline & $\mathrm{Zn}(\mathrm{OAc})_{2}$ & 3 & 252.9 & 30.8 & 55.0 & 10.7 \\
\hline & & 4 & 250.6 & 30.1 & 55.9 & 11.9 \\
\hline \multirow[t]{3}{*}{ Starting post-consumer PET } & & - & 398.8 & 79.5 & - & 15.9 \\
\hline & & 2 & 212.8 & 24.0 & 62.6 & 12.0 \\
\hline & TNT & 3 & 230.2 & 26.3 & 61.6 & 6.0 \\
\hline \multirow{4}{*}{ Post-consumer PET } & & 4 & 203.8 & 24.7 & 64.8 & 7.9 \\
\hline & & 2 & 232.0 & 26.4 & 57.0 & 13.4 \\
\hline & $\mathrm{Zn}(\mathrm{OAc})_{2}$ & 3 & 255.2 & 26.0 & 53.4 & 7.7 \\
\hline & & 4 & 225.7 & 26.8 & 59.6 & 11.3 \\
\hline
\end{tabular}

Ti 5\% w. 1. = initial temperature of decomposition ( $5 \%$ weight loss); W. $\mathrm{L}_{1}=$ weight loss $1 ; \mathrm{W} . \mathrm{L}_{2}=$ weight loss 2. 


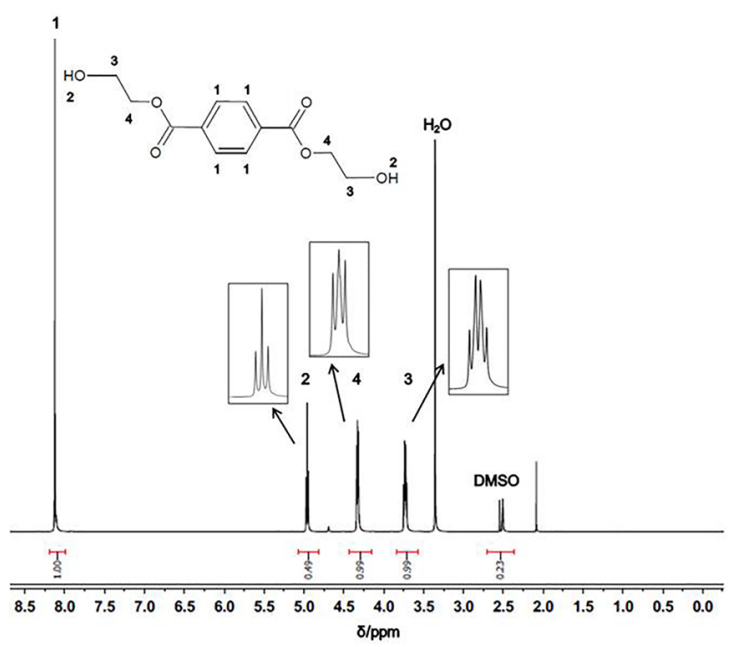

Figure 7. ${ }^{1} \mathrm{H}-\mathrm{NMR}$ spectrum for BHET, the main product of PET glycolysis.

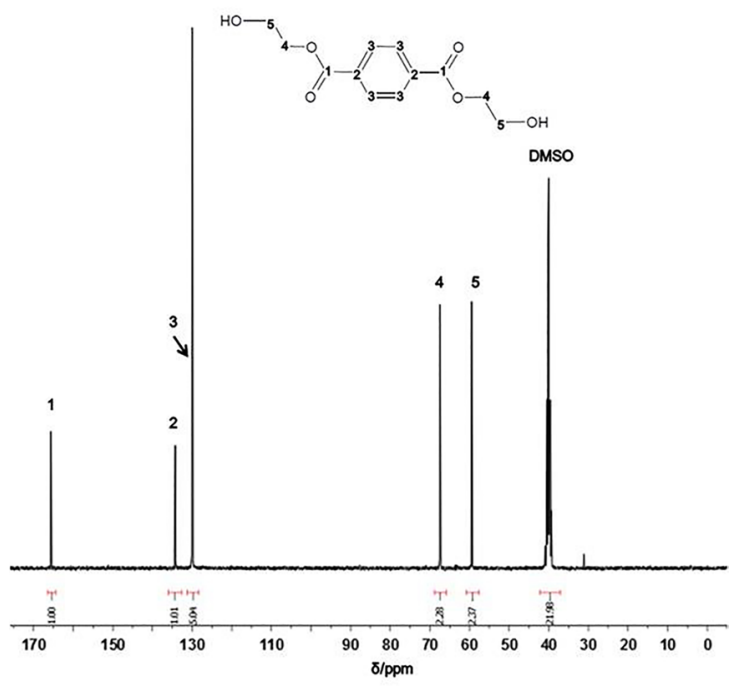

Figure 8. ${ }^{13} \mathrm{C}-\mathrm{NMR}$ spectrum for BHET, the main product of PET glycolysis.

$\left(-\mathrm{CH}_{2}-\right)$ adjacent to the $-\mathrm{COO}$ groups $\left(\delta_{\mathrm{H}}=4.33 \mathrm{ppm}, \mathrm{t}\right.$, $4 \mathrm{H})$, respectively. The sign around of $2.5 \mathrm{ppm}$ is DMSO and in 3.3 and $2.0 \mathrm{ppm}$ can be attributed to $\mathrm{H}_{2} \mathrm{O}$ residual and contamination. ${ }^{9,31,36}$ The ${ }^{13} \mathrm{C}$-NMR spectrum of BHET is shown in Figure 8 with chemical structure illustration.

The sign $1\left(\delta_{\mathrm{C}}=165.65 \mathrm{ppm}\right), 2\left(\delta_{\mathrm{C}}=134.24 \mathrm{ppm}\right), 3\left(\delta_{\mathrm{C}}\right.$ $=129.96 \mathrm{ppm}), 4\left(\delta_{\mathrm{C}}=67.48 \mathrm{ppm}\right)$ and $5\left(\delta_{\mathrm{C}}=59.48 \mathrm{ppm}\right)$ are assigned to the carbons of the chemical structure of BHET, as shown in Figure 8. The sign of DMSO appears in $40 \mathrm{ppm}$.

The yield values in $\%$ of BHET, turnover number (TON) and turnover frequency (TOF) are shown in Tables 2 (for virgin PET) and 3 (for post-consumer PET). TON and TOF are parameters that indicate the ability of a catalyst to convert reactants to products in molar units before becoming inactive and the number of cycles that this catalyst is able to make per time unit ${ }^{37}$, respectively.
Table 2. Yield, TON and TOF results obtained for BHET from virgin PET for different reaction times.

\begin{tabular}{lcccc}
\hline Catalyst & Time (h) & $\begin{array}{c}\text { BHET } \\
\text { yield (\%) }\end{array}$ & TON & TOF (h' $\left.\mathbf{h}^{-1}\right)$ \\
\hline \multirow{2}{*}{ TNT } & 2 & 81.3 & 383 & 191 \\
& 3 & 83.9 & 395 & 132 \\
& 4 & 80.2 & 377 & 94 \\
Zn(OAc) & 2 & 78.5 & 225 & 112 \\
& 3 & 79.4 & 227 & 76 \\
& 4 & 74.7 & 214 & 53 \\
\hline
\end{tabular}

Values of PET conversion for all reactions (using both virgin and post-consumer PET) were above 99\%. BHET yields were obtained above $70 \%$ using the $\mathrm{Zn}(\mathrm{OAc})_{2}$ catalyst and above $80 \%$ with the use of TNT for virgin PET (Table 2). However, the reaction time seems to have no significant effect on the BHET yields regardless the catalyst. The main difference is in the results of TON and TOF, where larger values of these catalytic parameters were obtained with TNT, proving itself as a most promising catalyst than the commercial catalyst. For both catalysts there were a decrease in the values of TOF with longer reaction times, indicating that in 2 hours of glycolysis obtains the highest catalytic efficiency (greater number of cycles per time), and thereby a high yield of BHET.

For reactions using post-consumer PET (Table 3 ) again TNT performance were more effective than zinc acetate, i.e. higher TON and TOF to TNT as compared with $\mathrm{Zn}(\mathrm{OAc})_{2}$, as noted in Table 2. BHET yields remains similar to all reaction times. When comparing values of TOF and the nature of PET it is observed that TNT were more effective when compared with $\mathrm{Zn}(\mathrm{OAc})_{2}$ in all reactions (virgin and post-consumer). Futhermore, TNT decrease their catalytic efficiency (Tables 2 and 3), but this isn't that significant. It can be attributed to additives present in post-consumer PET.

In view of these results, it has been observed that the optimal reaction time is 2 hours, because high yields and high values of TOF are achieved with minimal energy expenditure. Consequently, TNT are the most promising catalysts compared with zinc acetate in all reactions. The glycolysis reaction can occur via acyl-type $\left(\mathrm{A}_{\mathrm{AC}} 2\right)$ mechanism according to literature ${ }^{38}$. Different metallic-based catalysts are used in this reaction,

Table 3. Yield, TON and TOF results obtained for BHET from post-consumer PET for different reaction times.

\begin{tabular}{lcccc}
\hline Catalyst & Time (h) & $\begin{array}{c}\text { BHET } \\
\text { yield (\%) }\end{array}$ & TON & TOF $\left(\mathbf{h}^{-\mathbf{1}}\right)$ \\
\hline \multirow{2}{*}{ TNT } & 2 & 76.7 & 361 & 181 \\
& 3 & 73.2 & 345 & 115 \\
& 4 & 59.0 & 268 & 67 \\
Zn(OAc) & 2 & 82.6 & 237 & 118 \\
& 3 & 68.5 & 196 & 65 \\
& 4 & 81.8 & 228 & 57 \\
\hline
\end{tabular}


however depending of metal kind the mechanism will be different. When the catalyst is the $\mathrm{Zn}(\mathrm{OAc})_{2}$, the zinc atom acts as Lewis acid, interacting with oxygen from carbonyl group, corresponding to the ester group from PET chain, favoring nucleophilic attack of EG ${ }^{9}$. In the case of TNT as catalyst, a high actives sites quantity is available $\left(\mathrm{Na}^{+}\right.$ions and titanium atoms). Sodium ions will activate the nucleophile (EG) to attack the carbon from carbonyl group, corresponding to ester group while the titanium atoms produce a bidentate specie via coordination of the two oxygens of ester group of PET. Thus EG can attack more easily the carbon from carbonyl group that it is more susceptible. This behavior justify the higher activity of TNT when compared with $\mathrm{Zn}(\mathrm{OAc})_{2}$, making it the best catalyst for PET depolymerization by glycolysis.

\section{Conclusions}

Titanates nanotubes (TNT) were successfully synthesized by the hydrothermal method with $8.6 \mathrm{~nm} \pm 1.4 \mathrm{~nm}$ external diameter. They were applied as catalyst in reactions of glycolysis of PET depolymerization comparing the results with a commercial catalyst, zinc acetate. In the reactions using virgin PET, the TNT were promising having higher yields of BHET compared to the results obtained with $\mathrm{Zn}(\mathrm{OAc})_{2}$, and 2 hours reaction being more efficient. The obtainment of BHET was confirmed by ${ }^{1} \mathrm{H}$ and ${ }^{13} \mathrm{C}-\mathrm{NMR}$ analysis. Furthermore, the nature of PET influences decreasing the efficiency of TNT for glycolysis reaction when used post-consumer PET with BHET yields lower than those obtained $\mathrm{Zn}(\mathrm{OAc})_{2}$.

\section{Acknowledgements}

The authors thank CNPq and CAPES by scholarships. PUCRS and UFRGS for technical support. RL thanks CNPq for Technological Development-DT scholarship.

\section{References}

1. Eshaq G, Elmetwally AE. (Mg-Zn)-Al layered double hydroxide as a regenerable catalyst for the catalytic glycolysis of polyethylene terephthalate. Journal of Molecular Liquids. 2016;214:1-6.

2. Welle F. Twenty years of PET bottle to bottle recycling-An overview. Resources, Conservation and Recycling. 2011;55(11):865-875.

3. Bartolome L, Imran M, Cho BG, Al-Masry WA, Kim DH. Recent Developments in the Chemical Recycling of PET. In: Achilias D, ed. Material Recycling - Trends and Perspectives. Rijeka: InTech; 2012. p. 65-84.

4. Ghaemy M, Mossaddegh K. Depolymerisation of poly(ethylene terephthalate) fibre wastes using ethylene glycol. Polymer Degradation and Stability. 2005;90(3):570-576

5. Yue QF, Wang CX, Zhang LN, Ni Y, Jin YX. Glycolysis of poly(ethylene terephthalate) (PET) using basic ionic liquids as catalysts. Polymer Degradation and Stability. 2011;96(4):399403.
6. López-Fonseca R, Duque-Ingunza I, de Rivas B, Arnaiz S, Gutiérrez-Ortiz JI. Chemical recycling of post-consumer PET wastes by glycolysis in the presence of metal salts. Polymer Degradation and Stability. 2010;95(6):1022-1028.

7. Chen JW, Chen LW. The glycolysis of poly (ethylene terephthalate). Journal of Applied Polymer Science. 1999;73(1):35-40.

8. Troev K, Grancharov G, Tsevi R, Gitsov I. A novel catalyst for the glycolysis of poly(ethylene terephthalate). Journal of Applied Polymer Science. 2003;90(4):1148-1152.

9. Wang S, Wang C, Wang H, Chen X, Wang S. Sodium titanium tris(glycolate) as a catalyst for the chemical recycling of poly(ethylene terephthalate) via glycolysis and repolycondensation. Polymer Degradation and Stability. 2015;114:105-114.

10. Al-Sabagh AM, Yehia FZ, Eissa AMF, Moustafa ME, Eshaq G, Rabie $\mathrm{AM}$, et al. $\mathrm{Cu}$ - and $\mathrm{Zn}$-acetate-containing ionic liquids as catalysts for the glycolysis of poly(ethylene terephthalate). Polymer Degradation and Stability. 2014;110:364-377.

11. Ferreira HS, Rangel M do C. Nanotecnologia: aspectos gerais e potencial de aplicação em catálise. Química Nova. 2009;32(7):1860-1870.

12. Galetti AE, Barroso MN, Monzón A, Abello MC. Synthesis of Nickel Nanoparticles Supported on Carbon Using a Filter Paper as Biomorphic Pattern for Application in Catalysis. Materials Research. 2015;18(6):1278-1283.

13. Camargo PHC, Satyanarayana KG, Wypych F. Nanocomposites: synthesis, structure, properties and new application opportunities. Materials Research. 2009;12(1):1-39.

14. Camposeco R, Castillo S, Mejia-Centeno I, Navarrete J, Rodriguez-Gonzalez V. Behavior of Lewis and Brönsted surface acidity featured by $\mathrm{Ag}, \mathrm{Au}, \mathrm{Ce}, \mathrm{La}, \mathrm{Fe}, \mathrm{Mn}, \mathrm{Pd}, \mathrm{Pt}, \mathrm{V}$ and $\mathrm{W}$ decorated on protonated titanate nanotubes. Microporous and Mesoporous Materials. 2016;236:235-243.

15. Parayil SK, Razzaq A, Park SM, Kim HR, Grimes CA, In S Il. Photocatalytic conversion of $\mathrm{CO}_{2}$ to hydrocarbon fuel using carbon and nitrogen co-doped sodium titanate nanotubes. Applied Catalysis A: General. 2015;498:205-213.

16. Monteiro WF, Vieira MO, Aquino AS, de Souza MO, de Lima J, Einloft S, et al. $\mathrm{CO}_{2}$ conversion to propylene carbonate catalyzed by ionic liquid containing organosilane groups supported on titanate nanotubes/nanowires. Applied Catalysis A: General. 2017;544:46-54.

17. Hernández-Hipólito $P$, García-Castillejos M, Martínez-Klimova E, Juárez-Flores N, Gómez-Cortés A, Klimova TE. Biodiesel production with nanotubular sodium titanate as a catalyst. Catalysis Today. 2014;220-222:4-11.

18. Hernández-Hipólito $\mathrm{P}$, Juárez-Flores N, Martínez-Klimova E, Gómez-Cortés A, Bokhimi X, Escobar-Alarcón L, et al. Novel heterogeneous basic catalysts for biodiesel production: Sodium titanate nanotubes doped with potassium. Catalysis Today. 2015;250:187-196.

19. Salinas D, Guerrero S, Cross A, Araya P, Wolf EE. Potassium titanate for the production of biodiesel. Fuel. 2016;166:237-244.

20. Nepak D, Srinivas D. Spectroscopy and catalytic activity study of gold supported on barium titanate nanotubes for styrene epoxidation. Applied Catalysis A: General. 2016;523:61-72. 
21. Salmasi M, Fatemi S, Mortazavi Y. Fabrication of promoted $\mathrm{TiO} 2$ nanotubes with superior catalytic activity against $\mathrm{TiO}_{2}$ nanoparticles as the catalyst of oxi-desulfurization process. Journal of Industrial and Engineering Chemistry. 2016;39:6676.

22. Monteiro WF, dos Santos CAB, Einloft S, Oberson M, Carone CLP, Ligabue RA. Preparation of Modified Titanate Nanotubes and Its Application in Polyurethane Nanocomposites. Macromolecular Symposia. 2016;368(1):93-97.

23. Kasuga T, Hiramatsu M, Hoson A, Sekino T, Niihara K. Formation of Titanium Oxide Nanotube. Langmuir. 1998;14(12):31603163 .

24. Viana ME, Riul A, Carvalho GM, Rubira AF, Muniz EC. Chemical recycling of PET by catalyzed glycolysis: Kinetics of the heterogeneous reaction. Chemical Engineering Journal. 2011;173(1):210-219.

25. Xi G, Lu M, Sun C. Study on depolymerization of waste polyethylene terephthalate into monomer of bis(2-hydroxyethyl terephthalate). Polymer Degradation and Stability. 2005;87(1):117120.

26. Leong KH, Monash P, Ibrahim S, Saravanan P. Solar photocatalytic activity of anatase $\mathrm{TiO}_{2}$ nanocrystals synthesized by nonhydrolitic sol-gel method. Solar Energy. 2014;101:321-332.

27. Wang $\mathrm{H}$, Cao S, Fang Z, Yu F, Liu Y, Weng X, et al. $\mathrm{CeO}_{2}$ doped anatase $\mathrm{TiO}_{2}$ with exposed (001) high energy facets and its performance in selective catalytic reduction of $\mathrm{NO} \mathrm{NH}_{3}$. Applied Surface Science. 2015;330:245-252.

28. Wang L, Liu W, Wang T, Ni J. Highly efficient adsorption of $\mathrm{Cr}(\mathrm{VI})$ from aqueous solutions by amino-functionalized titanate nanotubes. Chemical Engineering Journal. 2013;225:153-163.

29. Liu J, Liu Y, Wu Z, Chen X, Wang H, Weng X. Polyethyleneimine functionalized protonated titanate nanotubes as superior carbon dioxide adsorbents. Journal of Colloid and Interface Science. 2012;386(1):392-397.
30. Thennarasu S, Rajasekar K, Balkis Ameen K. Hydrothermal temperature as a morphological control factor: Preparation, characterization and photocatalytic activity of titanate nanotubes and nanoribbons. Journal of Molecular Structure. 2013;1049:446457.

31. Imran M, Kim DH, Al-Masry WA, Mahmood A, Hassan A, Haider S, et al. Manganese-, cobalt-, and zinc-based mixedoxide spinels as novel catalysts for the chemical recycling of poly(ethylene terephthalate) via glycolysis. Polymer Degradation and Stability. 2013;98(4):904-915.

32. Awaja F, Pavel D. Recycling of PET. European Polymer Journal. 2005;41(7):1453-1477.

33. Kim H, Gilbert SG, Johnson JB. Determination of Potential Migrants from Commercial Amber Polyethylene Terephthalate Bottle Wall. Pharmaceutical Research. 1990;7(2):176-179.

34. Coltro L, Buratin AEP. Garrafas de PET para óleo comestível: avaliação da barreira à luz. Polímeros. 2004;14(3):206-211.

35. Geng Y, Dong T, Fang P, Zhou Q, Lu X, Zhang S. Fast and effective glycolysis of poly(ethylene terephthalate) catalyzed by polyoxometalate. Polymer Degradation and Stability. 2015;117:30-36.

36. Imran M, Kim BK, Han M, Cho BG, Kim DH. Sub-and supercritical glycolysis of polyethylene terephthalate (PET) into the monomer bis(2-hydroxyethyl) terephthalate (BHET). Polymer Degradation and Stability. 2010;95(9):1686-1693.

37. Umpierre AP, de Jesús E, Dupont J. Turnover Numbers and soluble Metal Nanoparticles. ChemCatChem Catalysis. 2011;3(9):1413-1418.

38. Otton J, Ratton S. Investigation of the formation of poly(ethylene terephthalate) with model molecules: Kinetics and mechanism of the catalytic esterification and alcoholysis reactions. I. Carboxylic acid catalysis (monofunctional reactants). Journal of Polymer Science Part A: Polymer Chemistry. 1988;26(8):2183-2197. 